\title{
What level of D-dimers can safely exclude pulmonary embolism in COVID-19 patients presenting to the emergency department?
}

\author{
Marie-Pierre Revel ${ }^{1,2}\left(\mathbb{D} \cdot\right.$ Nathanael Beeker $^{1,3} \cdot$ Raphael Porcher $^{1,4} \cdot$ Léa Jilet $^{3} \cdot$ Laure Fournier $^{1,5} \cdot$ Bastien Rance $^{1,6}$. \\ Guillaume Chassagnon $^{1,2} \cdot$ Michaela Fontenay ${ }^{1,7,8}$. Olivier Sanchez ${ }^{1,9,10} \cdot$ on behalf of the AP-HP /Universities/ \\ Inserm COVID-19 research collaboration, AP-HP Covid CDR Initiative
}

Received: 25 June 2021 / Revised: 14 September 2021 / Accepted: 30 September 2021 / Published online: 7 January 2022

(c) The Author(s), under exclusive licence to European Society of Radiology 2022

\begin{abstract}
Objectives To identify which level of D-dimer would allow the safe exclusion of pulmonary embolism (PE) in COVID-19 patients presenting to the emergency department (ED).

Methods This retrospective study was conducted on the COVID database of Assistance Publique - Hôpitaux de Paris (APHP). COVID-19 patients who presented at the ED of AP-HP hospitals between March 1 and May 15, 2020, and had CTPA following D-dimer dosage within $48 \mathrm{~h}$ of presentation were included. The D-dimer sensitivity, specificity, and positive and negative predictive values were calculated for different D-dimer thresholds, as well as the false-negative and failure rates, and the number of CTPAs potentially avoided.

Results A total of 781 patients (mean age 62.0 years, $53.8 \%$ men) with positive RT-PCR for SARS-Cov- 2 were included and 60 of them $(7.7 \%)$ had CTPA-confirmed PE. Their median D-dimer level was significantly higher than that of patients without PE $\left(4,013 \mathrm{vs} 1,198 \mathrm{ng} \cdot \mathrm{mL}^{-1}, p<0.001\right)$. Using $500 \mathrm{ng} \cdot \mathrm{mL}^{-1}$, or an age-adjusted cut-off for patients $>50$ years, the sensitivity and the NPV were above $90 \%$. With these thresholds, $17.1 \%$ and $31.5 \%$ of CTPAs could have been avoided, respectively. Four of the 178 patients who had a D-dimer below the age-adjusted cutoff had PE, leading to an acceptable failure rate of $2.2 \%$. Using higher D-dimer cut-offs could have avoided more CTPAs, but would have lowered the sensitivity and increased the failure rate. Conclusion The same D-Dimer thresholds as those validated in non-COVID outpatients should be used to safely rule out PE. Key Points

- The median D-dimer level was significantly higher in COVID-19 patients with PE as compared to those without PE (4,013 $\mathrm{ng} \cdot \mathrm{mL}^{-1}$ vs $1,198 \mathrm{ng} \cdot \mathrm{mL} \mathrm{L}^{-1}$ respectively, $\left.\mathrm{p}<0.001\right)$.

- Using $500 \mathrm{ng} \cdot \mathrm{mL}^{-1}$, or an age-adjusted D-dimer cut-off to exclude pulmonary embolism, the sensitivity and negative predictive value were above $90 \%$.

- Higher cut-offs would lead to a reduction in the sensitivity below $85 \%$ and an increase in the failure rate, especially for patients under 50 years.
\end{abstract}

Collaborators are listed in the Online supplementary material

Marie-Pierre Revel

marie-pierre.revel@aphp.fr ${ }^{1}$

Université de Paris, 75006 Paris, France

2 Radiology Department, Assistance Publique-Hôpitaux de Paris (AP-HP), Hôpital Cochin, Service de Radiologie27 rue du Faubourg Saint Jacques, 75014 Paris, France

3 Assistance Publique-Hôpitaux de Paris (AP-HP), Unité de Recherche Clinique, Hôpital Cochin, Paris, France

4 Assistance Publique-Hôpitaux de Paris (AP-HP), Centre d'épidémiologie clinique, Hôtel-Dieu, Paris, France

5 Assistance Publique-Hôpitaux de Paris (AP-HP), Service de Radiologie, Hôpital Européen, Georges Pompidou, Paris, France
6 Assistance Publique-Hôpitaux de Paris (AP-HP), Département d'Informatique Médicale, Biostatistiques Et Santé Publique, Hôpital Européen Georges Pompidou, Paris, France

7 Assistance Publique-Hôpitaux de Paris (AP-HP), Service de d'hématologie biologique, Hôpital Cochin, Paris, France

8 Institut Cochin INSERM U1016, CNRS UMR8104, Paris, France

9 Assistance Publique-Hôpitaux de Paris (AP-HP), Service de Pneumologie Et Soins Intensifs, Hôpital Européen, Georges Pompidou, INSERM UMRS-1140 Innovative Therapies in Hemostasis and Biosurgical Research Lab (Carpentier Foundation), Paris, France

10 F-CRIN INNOVTE, Saint-Etienne, France 
Keywords Pulmonary embolism · COVID-19 pandemic · Angiography, computed tomography · Pulmonary thromboembolism $\cdot$ Diagnostic technics and procedures

\begin{tabular}{|c|c|}
\hline \multicolumn{2}{|c|}{ Abbreviations } \\
\hline BMI & Body mass index \\
\hline COVID-19 & Coronavirus disease 2019 \\
\hline CTPA & $\begin{array}{l}\text { Computed tomography pulmonary } \\
\text { angiography }\end{array}$ \\
\hline ED & Emergency department \\
\hline ISTH & $\begin{array}{l}\text { International Society on Thrombosis and } \\
\text { Haemostasis }\end{array}$ \\
\hline OR & Odd ratio \\
\hline PE & Pulmonary embolism \\
\hline RT-PCR & $\begin{array}{l}\text { Reverse transcription-polymerase chain } \\
\text { reaction }\end{array}$ \\
\hline SARS-Cov-2 & $\begin{array}{l}\text { Severe acute respiratory } \\
\text { syndrome-coronavirus- } 2\end{array}$ \\
\hline & Venous thromboembolism \\
\hline
\end{tabular}

\section{Introduction}

Patients infected with SARS-Cov-2, developing COVID19 , are at risk of thromboembolic complications, due to the activation of coagulation. Severe COVID-19 cases are indeed associated with increased levels of inflammatory and prothrombotic biomarkers such as interleukin- 6 and increased levels of D-dimer [1]. Furthermore, it has been demonstrated that SARS-Cov-2 has the capacity to directly infect the endothelial cells and induce endotheliitis, which can also promote local thrombosis [2]. The awareness of an increased risk of pulmonary embolism (PE) has resulted from numerous articles reporting PE in COVID19 patients, with case reports followed by retrospective series [3-13].

However, the reported incidence varies widely, from less than 6\% at admission [13] to $30 \%$ when CT pulmonary angiography (CTPA) is performed both at initial evaluation and during follow-up [6]. A meta-analysis including 27 studies with 3,342 patients reported that PE was more frequently found in severely ill patients. The pooled incidence rates were $24.7 \%$ (95\% CI: $18.6,32.1$ ) in critically ill patients vs $10.5 \%$ (95\% CI: $5.1,20.2)$ for patients not admitted to the intensive care unit (ICU) [14]. Another meta-analysis based on 3,487 patients from 30 studies reported venous thromboembolism (VTE) in $24 \%$ of patients admitted to the ICU, whereas VTE affected only $9 \%$ of patients in normal wards [15]. A third metaanalysis including 49 studies with 18,093 patients reported a pooled PE incidence of 7.1\% (95\%CI, 5.3-9.1) and confirmed that PE was more frequently diagnosed in ICU patients as opposed to in the wards $(27.9 \%$ vs $7.1 \%)$ [16].
There is no current recommendation to routinely perform a CTPA when evaluating COVID-19 patients, with the risk to miss PE when performing unenhanced CT. The complexity of the assessment is all the greater as the symptoms of COVID pneumonia and PE strongly overlap, both of which are responsible for dyspnea and/or desaturation.

Established guidelines exist for managing outpatients with PE suspicion [17], based on clinical probability assessment and D-dimer dosage. Outcome studies have shown that the 3 -month thromboembolic risk is $<1 \%$ in patients with low or intermediate clinical probability and D-dimer $<500 \mathrm{ng} \cdot \mathrm{mL}^{-1}$ who are left untreated [18]. The adjust-PE study has demonstrated that the D-dimer level adjusted to patient age, with higher thresholds in older patients (age $\times 10 \mathrm{ng} \cdot \mathrm{mL}^{-1}$ ), can safely rule out PE [19].

However, these guidelines might not be suitable for COVID-19 patients, one reason being that estimating the clinical probability of PE is complex in patients with COVID-19 pneumonia. Of note, risk factors such as history of previous thrombosis or active malignancy are not reported to be significantly different between $P E$ positive and PE negative COVID-19 patients [20, 21].

Thus, the objective of this retrospective study was to evaluate the optimal D-dimer levels that might safely exclude acute PE independently from the pre-test clinical probability in COVID-19 patients presenting to the emergency department.

\section{Material and methods}

This retrospective study was approved by the Scientific and Ethical Committee of Assistance Publique - Hôpitaux de Paris (AP-HP) (IRB authorization number 00011591). Patients included in the study did not oppose to the re-use of their data.

\section{Data source}

Assistance Publique - Hôpitaux de Paris (AP-HP) is a group of 39 hospitals $(22,474$ beds) mainly located in the Greater Paris area with 1.5 million hospitalizations per year (10\% of all hospitalizations in France). Since 2014, AP-HP has been building a data warehouse (Entrepôt de Données de Santé, EDS) based on a clinical data repository (CDR), aggregating day-to-day clinical data from 8.8 million patients [22]. From the beginning of the COVID-19 pandemic, the EDS-COVID database retrieved electronic health records from all AP-HP hospitals and aggregated 
them into a clinical data warehouse following Observational Medical Outcomes Partnership (OMOP) common data model [23]. The analysis conducted for this study followed the recommendations provided by the REporting of studies Conducted using Observational Routinely-collected health Data (RECORD) Statement [24].

\section{Study population}

Eligible patients were those with a positive reverse transcription-polymerase chain reaction (RT-PCR) result on the nasopharyngeal swab for SARS-Cov-2 who presented to the emergency department (ED) of one of the AP-HP hospitals between March 1 and May 15, 2020, because of respiratory symptoms. Among them, patients who had a D-dimer dosage and underwent a CTPA within $48 \mathrm{~h}$ from the presentation at the ED were included. Patients were classified as PE positive based on the International Classification of Disease 10th Revision (ICD-10) code specific for acute PE with or without acute cor pulmonale (I26) and the conclusion of the CTPA reports. The reports were in a structured format; otherwise, they were retrieved for confirmation or exclusion of PE. Patients with an indeterminate CTPA result or an unavailable CT report were excluded.

\section{D-dimer test}

D-dimer testing was measured using a locally available quantitative and highly sensitive D-dimer assay. One of the following methods was used: ELISA VIDAS ${ }^{\circledR}$ D-Dimer Exclusion $^{\mathrm{TM}}$ II (bioMérieux SA) in 160 patients, or automated latex-enhanced turbidimetric immunoassays: STA®Liatest ${ }^{\circledR}$ D-Di Plus (Diagnostica Stago) in 475 patients, and HemosIL D-dimer HS500® (Instrumentation Laboratories) in 135 patients. The technique used for D-dimer dosage could not be retrieved for 6 patients.

\section{Statistical analysis}

Continuous data is presented as mean \pm standard deviation or median and interquartile range as appropriate, while categorical data is summarized as counts and percentages.

The Wilcoxon-Mann-Whitney, Student $t$ test, and Fischer exact test were used for comparisons. Receiver operating characteristic (ROC) curve analysis was conducted to evaluate the diagnostic performance of D-dimer, evaluated by the area under the curve (AUC), with CTPA as the reference standard. This was performed for the whole study sample as well as for patients up to and over the age of 50 years. We calculated the sensitivity, specificity, and positive and negative predictive values, with their 95\% confidence interval for different D-dimer thresholds, as well as the rate of false negatives, the failure rate, and the number of CTPAs which could have been avoided if the corresponding threshold had been used. The false-negative rate was defined as the number of patients with PE diagnosed by CTPA below the threshold considered divided by the total number of patients. The failure rate for each threshold was the proportion of patients with PE having a D-dimer value below the threshold considered among the subset of patients with a D-dimer value below this threshold.

All statistical analyses were performed with Spark-R software, version 2.4.2, on a Jupyter platform. A $p$ value of $\leq 0.05$ was considered significant for all statistical tests conducted.

\section{Results}

During the study period, 7,452 patients with SARS-Cov-2 infection confirmed by RT-PCR presented at the ED of AP-HP hospitals and D-dimer dosage was performed for 2,272 of them. Of these, 781 patients had conclusive CTPA results obtained within $24 \mathrm{~h}$ of D-dimer dosage and composed the study sample (flow chart, Fig. 1). The patient characteristics are presented in Table 1. Among preexisting comorbidities, hypertension was the most frequently reported at $19.7 \%$ of patients. A PE was diagnosed on CTPA for 60 patients, resulting in an overall PE prevalence of 7.7\% (5.9-9.8) at presentation in the ED. Patients with PE were more frequently of the male gender. Comorbidities were not significantly different between patients with and without PE.

The median D-dimer level was $1,269 \mathrm{ng} \cdot \mathrm{mL}^{-1}$ in the overall population and was significantly higher in patients with PE as compared to those without PE $\left(4,013 \mathrm{ng} \cdot \mathrm{mL}^{-1}\right.$ vs $1,198 \mathrm{ng} \cdot \mathrm{mL}^{-1}$ respectively, $\left.p<0.001\right)$. Most patients had signs of systemic inflammation with a median C-reactive protein (CRP) value of $67 \mathrm{mg} \cdot \mathrm{L}^{-1}$. Even though CRP values were higher in patients with PE, the CRP to $\mathrm{D}$-dimer ratio was lower, meaning that the increase of D-dimer was disproportionate (Table 1).

Regarding the diagnostic performance of D-dimer in the study sample, the area under the curve (AUC) was $0.814(95 \% \mathrm{CI}, 0.754,0.873)$ in the overall population, $0.834(95 \% \mathrm{CI}, 0.725,0.944)$ for patients up to 50 years, and $0.810(95 \% \mathrm{CI}, 0.740,0.881)$ for patients over 50 years (Fig. 2). The sensitivity, specificity, and predictive values of D-dimer at different thresholds are presented in Table 2, together with the false-negative rate, the failure rate, and the number of CTPAs which could have been avoided. Using the current validated thresholds of D-dimer in non-COVID-19 outpatients with PE suspicion (i.e., $500 \mathrm{ng} \cdot \mathrm{mL}^{-1}$, or an age-adjusted cut-off for patients $>50$ years), the sensitivity and the NPV were above $90 \%$ with low specificity. Using the fixed conventional cut-off $\left(<500 \mathrm{ng} \cdot \mathrm{mL}^{-1}\right)$ for patients $\leq 50$ years, $37 / 216$ patients $(17.1 \%)$ had negative $\mathrm{D}$-dimer and could 
Fig. 1 Flow chart

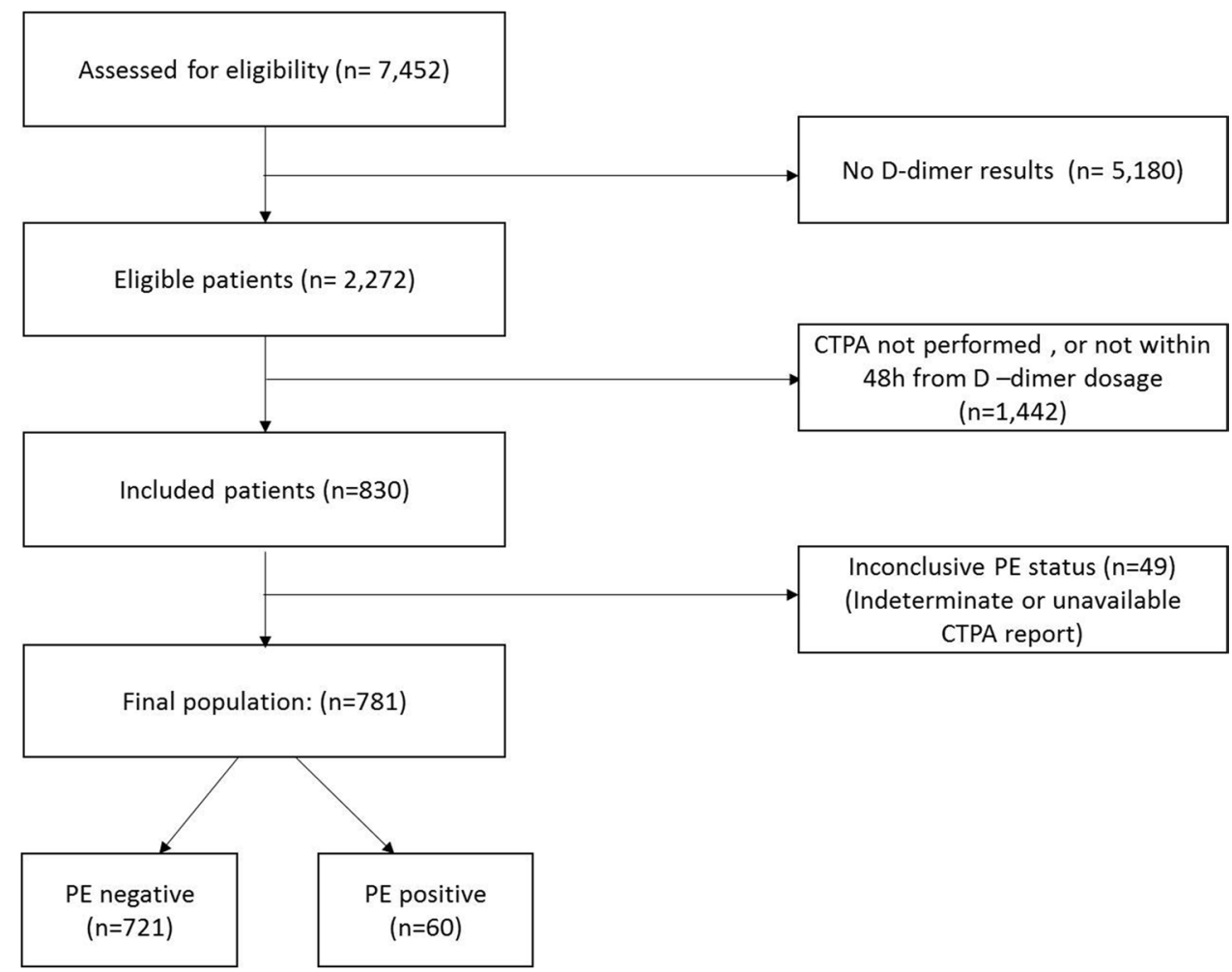

have avoided CTPA; none of them had PE. Using the ageadjusted cut-off for patients $>50$ years, $178 / 565$ patients (31.5\%) had a negative D-dimer and could have avoided CTPA. Among them, 4 had a PE leading to a failure rate of $2.2 \%$. Higher D-dimer cut-offs lowered the sensitivity and increased the specificity and the number of CTPAs which could have been avoided but at the cost of lower sensitivity and higher failure rates (Table 2).

\section{Discussion}

This large retrospective study supports using the same D-dimer thresholds as those validated in the general population to safely rule out PE in COVID-19 patients presenting to the ED. Using the fixed conventional cut-off value $\left(<500 \mathrm{ng} \cdot \mathrm{mL}^{-1}\right)$ up to 50 years and an age-adjusted D-dimer after 50 years would have avoided $17.1 \%$ and $31.5 \%$

Table 1 Characteristics of the study sample

\begin{tabular}{|c|c|c|c|c|}
\hline Characteristic & All $(n=781)$ & No PE $(n=721)$ & $\operatorname{PE}(n=60)$ & $p$ value \\
\hline Male sex $(n, \%)$ & $420(53.8)$ & $379(52.6)$ & $41(68.3)$ & 0.03 \\
\hline Age (years) mean (SD) & $62.0(17.6)$ & $61.9(17.7)$ & $63.3(16.2)$ & 0.53 \\
\hline$>50$ years $(n, \%)$ & $565(72.3)$ & $520(72.1)$ & $45(75.0)$ & 0.74 \\
\hline Body mass index $\geq 30.0 \mathrm{~kg} / \mathrm{m}^{2}$ & $92(11.8)$ & $86(11.9)$ & $6(10.0)$ & 0.81 \\
\hline Hypertension $(n, \%)$ & $154(19.7)$ & $147(20.4)$ & $7(11.7)$ & 0.14 \\
\hline Diabetes $(n, \%)$ & $95(12.2)$ & $91(12.6)$ & $4(6.7)$ & 0.14 \\
\hline Heart failure $(n, \%)$ & $42(5.4)$ & $40(5.5)$ & $2(3.3)$ & 0.66 \\
\hline Chronic kidney disease $(n, \%)$ & $25(3.2)$ & $22(3.1)$ & $3(5.0)$ & 0.66 \\
\hline Admitted in normal wards $(n, \%)$ & $437(56.0)$ & $400(55.5)$ & $37(61.7)$ & 0.43 \\
\hline Admitted to ICU $(n, \%)$ & $94(12.0)$ & $84(11.7)$ & $10(16.7)$ & 0.35 \\
\hline Deceased $(n, \%)$ & $105(13.4)$ & $96(13.3)$ & $9(15.0)$ & 0.86 \\
\hline D-dimer (ng.mL $\left.{ }^{-1}\right)$, median (IQR) & $1,269(759-2,228)$ & $1,198(730-1,966)$ & $4,013(2,279-5,378)$ & $<0.001$ \\
\hline C-reactive protein $\left(\mathrm{mg} . \mathrm{L}^{-1}\right)$, median (IQR) & $67(22-92)(n=748)$ & $63(21-88)(n=689)$ & $122(42-133)(n=59)$ & $<0.001$ \\
\hline $\begin{array}{l}\text { C-reactive protein to D-dimer ratio, median } \\
(\mathrm{IQR})(\times 100)\end{array}$ & $4.4(1.3-7.5)(n=748)$ & $4.6(1.4-7.7)(n=689)$ & $2.2(1.0-4.5)(n=59)$ & 0.002 \\
\hline
\end{tabular}


Fig. 2 ROC curve analysis of D-dimer diagnostic performance

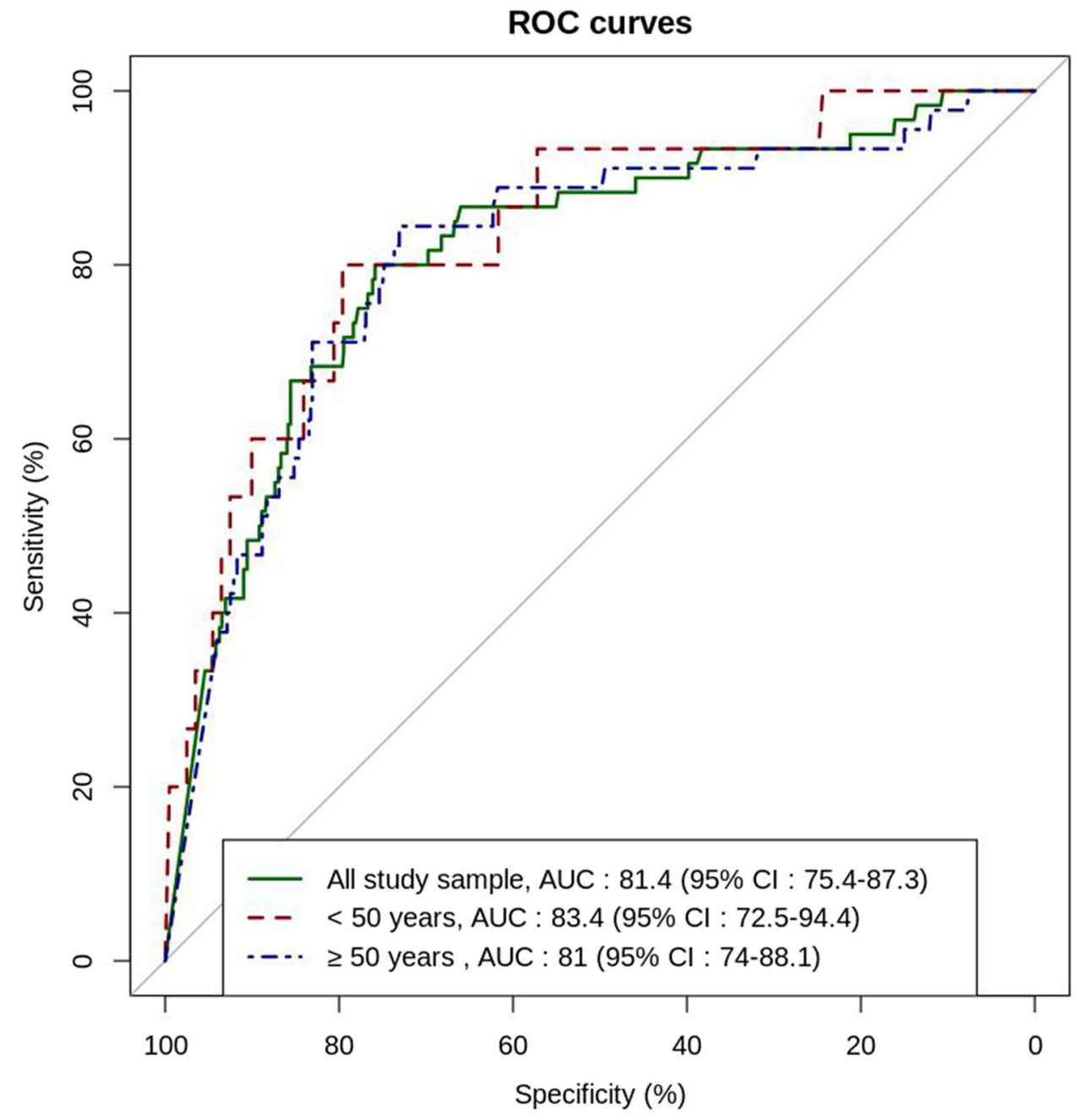

of CTPAs respectively, with a low rate of false negatives. Higher D-dimer thresholds would increase the number of CTPAs avoided but also the failure rates, especially for patients under the age of 50 years.

We focused our analysis on patients presenting to the ED because the usefulness of D-dimer to rule out PE is mainly validated for this category of patients [17]. Moreover, even though PE is mainly reported in severe COVID-19 patients admitted in ICU, the $7.7 \%$ prevalence observed in our study is not negligible and is in line with other reports, where PE prevalence in outpatients diagnosed with COVID-19 ranged from 5.7 to $14.2 \%[13,25]$. Suspecting PE in these patients is challenging because they share clinical characteristics with PE and many of them have increased D-Dimer levels due to systemic inflammation, leading to a difficult CTPA prescription decision. Since the start of the pandemic and through the successive waves, physicians have become aware of the increased risk of PE associated with SARS-Cov-2 infection and have lowered their clinical threshold of PE suspicion in COVID patients admitted to the ED, resulting in a substantial increase in CTPA prescribing. Data from the French National Hospital Discharge database showed that $34.4 \%$ of Covid-19 patients presenting to the ED in March-April 2021 had CTPA, compared to $12.5 \%$ during the same time period in 2020. Moreover, D-dimer levels have a prognostic value for in-hospital mortality, the reason why they are now almost systematically prescribed at ED admission [26]. This is likely to greatly increase the referral for CTPA of COVID-19 patients. A high D-dimer level was cited in $44 \%$ of the clinical information provided for CPTA imaging requests, in the study by Ooi et al. [11]. There are several reasons why avoiding CTPA, or at least rationalizing its prescription in COVID-19 patients, would be important. The administration of iodinated contrast medium can be problematic in patients at risk of developing acute kidney injury, which is the case for COVID-19 patients [27]. Contrast administration implies a closer contact between the infected patients and the technologists, with therefore a risk of contamination, which must be prevented by reinforced protective personal equipment. Lastly, increased DNA damage in circulating blood cells and increased organ doses are reported for X-ray exposures enhanced by iodinated contrast 


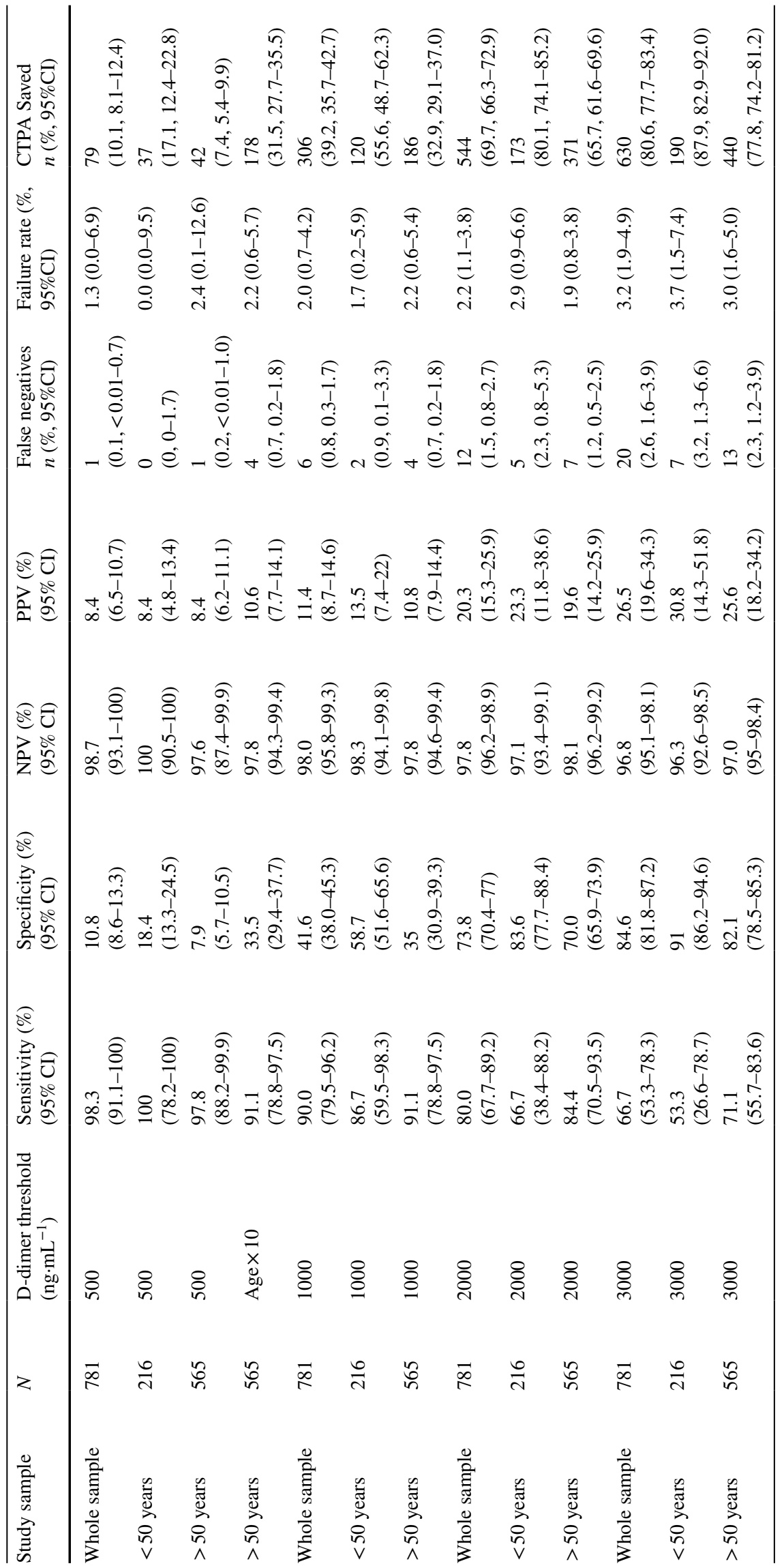


media, even though there is no evidence that this implies an increased risk of developing cancer [28]. However, none of these 3 risks justifies prohibiting performing CTPA, whereas the risk of dying from an untreated pulmonary embolism is at least 5\% [29]. Additionally, the use of contrast with a dual-energy technique offers the possibility to evaluate lung perfusion, which can be compromised due to microvascular thrombosis [30].

In the absence of a currently validated pre-test probability score for the COVID-19 outpatient population, our objective was to assess the D-dimer cut-off safely excluding PE independently from the pre-test clinical probability, taking into account the relatively low PE prevalence in such patients.

Different automated, well-validated, highly-sensitive, quantitative D-dimer assays were used in our study. Their results were pooled as it was already done in other recent diagnostic PE studies $[19,31]$. Our results suggest that the diagnostic performance of D-dimer for the exclusion of $\mathrm{PE}$ in COVID-19 patients at the ED is close to that reported in non-COVID outpatients [32]. The same validated D-dimer thresholds, either a fixed cut-off value of $500 \mathrm{ng} \cdot \mathrm{mL}^{-1}$ or an age-adjusted cut-off, can safely exclude PE with a low failure rate, close to the maximum acceptable failure rate according to the International Society on Thrombosis and Haemostasis (ISTH) recommendation [33].

Other D-dimer cut-off values have been suggested in the recent COVID-19 literature. A different approach was used which was to find the best compromise between sensitivity and specificity, not to minimize the risk of false negatives. Mouhat et al. suggested an optimal D-dimer cut-off value of $2,590 \mathrm{ng} \cdot \mathrm{mL}^{-1}$ to predict CTPA-confirmed PE in a retrospective series of 162 COVID-19 patients [21]. PE prevalence was much higher, reaching $27 \%$ in this series including $70 \%$ of inpatients. In another retrospective study based on 242 in- and outpatients where PE prevalence was $30 \%$, Ventura-Diaz et al. concluded that the optimal D-dimer cut-off to predict PE was 2,903 ng.mL $\mathrm{mL}^{-1}$ [34]. With this cut-off, the sensitivity was $81 \%$, and 98 CTPAs could have been avoided, but 13 PEs would have been missed. A third retrospective study based on 84 COVID-19 patients of whom $38 \%$ were PE positive proposed a D-dimer cut-off of $2,247 \mathrm{ng} \cdot \mathrm{mL}^{-1}$, providing $72 \%$ sensitivity and $74 \%$ specificity [11]. Compared to these three studies, the originality of our study, based on a larger study sample, was to specifically deal with outpatients. In our population, using D-dimer cutoffs such as those reported in these high prevalent PE studies would lead to a failure rate above the maximum acceptable value according to the ISTH recommendation [33].

Furthermore, as outlined by Korevaar and van Es in their commentary of [21], selecting the positivity threshold for a biomarker based on the highest Youden's index may be statistically meaningful, but clinically irrelevant, with an unacceptable rate of missed PEs [35].
Garcia-Olivé et al. developed a model for predicting PE depending on D-dimer levels and the number of days elapsed since COVID-19 diagnosis [36]. The AUC of the model was $0.80(0.70-0.90)$ before the age of 65 years but only $0.58(0.39-0.77)$ for older female patients. Regarding other risk factors, there are conflicting results regarding obesity, with BMI $>30 \mathrm{~kg} / \mathrm{m}^{2}$ being significantly more frequent for PE positive COVID-19 patients in some reports [21], whereas it was not in others $[6,20]$. None of the pre-existing comorbidities listed in our patient population, including obesity, was significantly different between PE positive and PE negative patients. PE positive patients were more frequently of male gender in our study, which may be related to the development of more severe forms of COVID-19 in men, with higher levels of inflammation and release of prothrombotic factors.

There are several limitations to our study. Firstly, a selection bias is likely present, since not all COVID-19 patients presenting to the ED with respiratory symptoms had both D-dimer and CTPA systematically performed. In particular, 1,442 patients with D-dimer had no CTPA within $24 \mathrm{~h}$ of dosage. There were no established guidelines across the different centers regarding when to perform CTPA or when to perform D-dimer assay, and the use of these tests was probably different among the different EDs. Our result should therefore not be interpreted as evaluating the diagnostic performance of D-dimer for PE in COVID-19 patients presenting to the ED with respiratory symptoms. Rather, it specifically addresses the question of whether a different D-dimer threshold than recommended outside COVID-19 would safely rule out PE and save unnecessary CTPAs. Secondly, central reading of CTPA studies was not performed to confirm or exclude PE. PE diagnosis relied on the conclusion of CTPA reports. However, a good inter-reader agreement is reported for the interpretation of CTPAs, with a unanimous agreement in $91 \%$ of cases [37]. Thirdly, the assay used to measure the level of D-dimer could not be identified for 6 patients of the study sample, and not all assays have the same sensitivity. However, this is unlikely to have influenced our results since highly sensitive assays were used for all other patients, representing $98.6 \%$ of the study sample. Lastly, there were only 216 patients at or under the age of 50 years in this study, which limited the possibility to better evaluate $\mathrm{D}$-dimer thresholds in this subgroup of patients.

In conclusion, our study supports using the same D-Dimer thresholds as those validated in the general population to safely rule out PE in COVID-19 patients presenting to the ED. Applying an age-adjusted threshold could save one-third of CTPAs in patients over 50 years, who represent the majority of COVID-19 patients. 
Supplementary Information The online version contains supplementary material available at https://doi.org/10.1007/s00330-021-08377-9.

Acknowledgements The authors thank the EDS AP-HP Covid consortium for integrating the AP-HP Health Data Warehouse team as well as all the AP-HP staff and volunteers who contributed to the implementation of the EDS-Covid database and operating solutions for this database. They also thank Dominic De La O for editorial assistance.

Funding The authors state that this work has not received any funding.

\section{Declarations}

Guarantor The scientific guarantor of this publication is Pr Mariepierre REVEL.

Conflict of interest The authors of this manuscript declare no relationships with any companies whose products or services may be related to the subject matter of the article.

Statistics and biometry One of the authors has significant statistical expertise.

Informed consent Written informed consent was waived by the Institutional Review Board.

Ethical approval Institutional Review Board approval was obtained.

\section{Methodology}

- retrospective

- COHORT study

- multicenter study

\section{References}

1. Lopez-Castaneda S, García-Larragoiti N, Cano-Mendez A et al (2021) Inflammatory and prothrombotic biomarkers associated with the severity of COVID-19 infection. Clin Appl Thromb 27:107602962199909. https://doi.org/10.1177/1076029621 999099

2. Varga Z, Flammer AJ, Steiger P et al (2020) Endothelial cell infection and endotheliitis in COVID-19. Lancet 395:1417-1418. https://doi.org/10.1016/S0140-6736(20)30937-5

3. Danzi GB, Loffi M, Galeazzi G, Gherbesi E (2020) Acute pulmonary embolism and COVID-19 pneumonia: a random association? Eur Heart J 41:1858-1858. https://doi.org/10.1093/eurhe artj/ehaa254

4. Xie Y, Wang X, Yang P, Zhang S (2020) COVID-19 complicated by acute pulmonary embolism. Radiol Cardiothorac Imaging 2:e200067. https://doi.org/10.1148/ryct.2020200067

5. Grillet F, Behr J, Calame P et al (2020) Acute pulmonary embolism associated with COVID-19 pneumonia detected with pulmonary CT angiography. Radiology 296:E186-E188. https://doi.org/ 10.1148/radiol.2020201544

6. Léonard-Lorant I, Delabranche X, Séverac F et al (2020) Acute pulmonary embolism in patients with COVID-19 at CT angiography and relationship to D -dimer levels. Radiology 296:E189E191. https://doi.org/10.1148/radiol.2020201561

7. Cui S, Chen S, Li X et al (2020) Prevalence of venous thromboembolism in patients with severe novel coronavirus pneumonia.
J Thromb Haemost 18:1421-1424. https://doi.org/10.1111/jth. 14830

8. Bompard F, Monnier H, Saab I et al (2020) Pulmonary embolism in patients with COVID-19 pneumonia. Eur Respir J 56:2001365. https://doi.org/10.1183/13993003.01365-2020

9. Alonso-Fernández A, Toledo-Pons N, Cosío BG et al (2020) Prevalence of pulmonary embolism in patients with COVID-19 pneumonia and high D-dimer values: a prospective study. PLoS One 15:e0238216. https://doi.org/10.1371/journal.pone.0238216

10. Klok FA, Kruip MJHA, van der Meer NJM et al (2020) Incidence of thrombotic complications in critically ill ICU patients with COVID19. Thromb Res. https://doi.org/10.1016/j.thromres.2020.04.013

11. Ooi MWX, Rajai A, Patel R et al (2020) Pulmonary thromboembolic disease in COVID-19 patients on CT pulmonary angiography - prevalence, pattern of disease and relationship to D-dimer. Eur J Radiol 132:109336. https://doi.org/10.1016/j.ejrad.2020.109336

12. Poyiadji N, Cormier P, Patel PY et al (2020) Acute pulmonary embolism and COVID-19. Radiology 297:E335-E338. https://doi. org/10.1148/radiol.2020201955

13. Jalaber C, Revel M-P, Chassagnon G et al (2020) Role of upfront CT pulmonary angiography at admission in COVID-19 patients. Thromb Res 196:138-140. https://doi.org/10.1016/j.thromres.2020. 08.037

14. Suh YJ, Hong H, Ohana M et al (2021) Pulmonary embolism and deep vein thrombosis in COVID-19: a systematic review and metaanalysis. Radiology 298:E70-E80. https://doi.org/10.1148/radiol. 2020203557

15. Porfidia A, Valeriani E, Pola R et al (2020) Venous thromboembolism in patients with COVID-19: systematic review and metaanalysis. Thromb Res 196:67-74. https://doi.org/10.1016/j.throm res.2020.08.020

16. Jiménez D, García-Sanchez A, Rali P et al (2021) Incidence of VTE and bleeding among hospitalized patients with coronavirus disease 2019. Chest 159:1182-1196. https://doi.org/10.1016/j.chest.2020. 11.005

17. Konstantinides SV, Meyer G, Becattini C et al (2019) 2019 ESC Guidelines for the diagnosis and management of acute pulmonary embolism developed in collaboration with the European Respiratory Society (ERS): the Task Force for the diagnosis and management of acute pulmonary embolism of the European Society of Cardiology (ESC). Eur Respir J 54:1901647. https://doi.org/10.1183/13993003. 01647-2019

18. Carrier M, Righini M, Djurabi RK et al (2009) VIDAS D-dimer in combination with clinical pre-test probability to rule out pulmonary embolism. A systematic review of management outcome studies. Thromb Haemost 101:886-892

19. Righini M, Van Es J, Den Exter PL et al (2014) Age-Adjusted D-Dimer Cutoff Levels to Rule Out Pulmonary Embolism: The ADJUST-PE Study. JAMA 311:1117. https://doi.org/10.1001/jama. 2014.2135

20. Infanta Leonor Thrombosis Research Group, Mestre-Gómez B, Lorente-Ramos RM et al (2021) Incidence of pulmonary embolism in non-critically ill COVID-19 patients. Predicting factors for a challenging diagnosis. J Thromb Thrombolysis 51:40-46. https://doi. org/10.1007/s11239-020-02190-9

21. Mouhat B, Besutti M, Bouiller K et al (2020) Elevated D-dimers and lack of anticoagulation predict $\mathrm{PE}$ in severe COVID-19 patients. Eur Respir J 56:2001811. https://doi.org/10.1183/13993003.01811-2020

22. Daniel C, Serre P, Orlova N, Bréant S, Paris N,Griffon N (2019) Initializing a hospital-wide data quality program. The AP-HP experience. Comput Methods Programs Biomed 181:104804. https://doi. org/10.1016/j.cmpb.2018.10.016

23. Hripcsak G, Duke JD, Shah NH et al (2015) Observational Health Data Sciences and Informatics (OHDSI): opportunities for observational researchers. Stud Health Technol Inform 216:574-578 
24. Benchimol EI, Smeeth L, Guttmann A et al (2015) The Reporting of studies Conducted using Observational Routinely-collected health Data (RECORD) statement. PLoS Med 12:e1001885. https://doi. org/10.1371/journal.pmed.1001885

25. Jevnikar M, Sanchez O, Chocron R et al (2021) Prevalence of pulmonary embolism in patients with COVID 19 at the time of hospital admission. Eur Respir J. https://doi.org/10.1183/13993003. 00116-2021

26. Chocron R, Duceau B, Gendron N et al (2021) D-dimer at hospital admission for COVID-19 are associated with in-hospital mortality, independent of venous thromboembolism: insights from a French multicenter cohort study. Arch Cardiovasc Dis. https://doi.org/10. 1016/j.acvd.2021.02.003

27. Hirsch JS, Ng JH, Ross DW et al (2020) Acute kidney injury in patients hospitalized with COVID-19. Kidney Int 98:209-218. https://doi.org/10.1016/j.kint.2020.05.006

28. Harbron R, Ainsbury EA, Bouffler SD et al (2017) Enhanced radiation dose and DNA damage associated with iodinated contrast media in diagnostic X-ray imaging. Br J Radiol 90:20170028. https://doi. org/10.1259/bjr.20170028

29. Stein PD, Chenevert TL, Fowler SE et al (2010) Gadoliniumenhanced magnetic resonance angiography for pulmonary embolism: a multicenter prospective study (PIOPED III). Ann Intern Med 152(434-443):W142-143. https://doi.org/10.7326/0003-4819-1527-201004060-00008

30. Patel BV, Arachchillage DJ, Ridge CA et al (2020) Pulmonary angiopathy in severe COVID-19: physiologic, imaging, and hematologic observations. Am J Respir Crit Care Med 202:690-699. https://doi. org/10.1164/rccm.202004-1412OC

31. Roy P-M, Friou E, Germeau B et al (2021) Derivation and validation of a 4-level clinical pretest probability score for suspected pulmonary embolism to safely decrease imaging testing. JAMA Cardiol. https://doi.org/10.1001/jamacardio.2021.0064

32. Di Nisio M, Squizzato A, Rutjes AWS et al (2007) Diagnostic accuracy of D-dimer test for exclusion of venous thromboembolism: a systematic review. J Thromb Haemost 5:296-304. https://doi.org/ 10.1111/j.1538-7836.2007.02328.x

33. Dronkers CEA, van der Hulle T, Le Gal G et al (2017) Towards a tailored diagnostic standard for future diagnostic studies in pulmonary embolism: communication from the SSC of the ISTH. J Thromb Haemost 15:1040-1043. https://doi.org/10.1111/jth.13654

34. Ventura-Díaz S, Quintana-Pérez JV, Gil-Boronat A et al (2020) A higher D-dimer threshold for predicting pulmonary embolism in patients with COVID-19: a retrospective study. Emerg Radiol 27:679-689. https://doi.org/10.1007/s10140-020-01859-1

35. Korevaar DA, van Es J (2021) Pulmonary embolism in COVID-19: D-dimer threshold selection should not be based on maximising Youden's index. Eur Respir J 57:2004279. https://doi.org/10.1183/ 13993003.04279-2020

36. Garcia-Olivé I, Sintes H, Radua J et al (2021) Predicting pulmonary embolism in patients infected with COVID-19 based on D-dimer levels and days between diagnosis of the infection and D-dimer determination. Monaldi Arch Chest Dis. https://doi.org/10.4081/ monaldi.2021.1622

37. Diffin DC, Leyendecker JR, Johnson SP et al (1998) Effect of anatomic distribution of pulmonary emboli on interobserver agreement in the interpretation of pulmonary angiography. AJR Am J Roentgenol 171:1085-1089. https://doi.org/10.2214/ajr.171.4.9763002

Publisher's note Springer Nature remains neutral with regard to jurisdictional claims in published maps and institutional affiliations. 